\title{
Proposal for the use of echocardiography in bloodstream infections due to different streptococcal species
}

Sandra Chamat-Hedemand ${ }^{1,2^{*}}$, Niels Eske Bruun ${ }^{1,3,4}$, Lauge Østergaard ${ }^{5}$, Magnus Arpi ${ }^{6}$, Emil Fosbøl ${ }^{5}$, Jonas Boel ${ }^{6}$, Louise Bruun Oestergaard ${ }^{2}$, Trine K. Lauridsen², Gunnar Gislason ${ }^{2,7}$, Christian Torp-Pedersen ${ }^{8,9}$ and Anders Dahl ${ }^{2}$

\begin{abstract}
Background: Infective endocarditis (IE) is diagnosed in 7-8\% of streptococcal bloodstream infections (BSIs), yet it is unclear when to perform transthoracic (TTE) and transoesophageal echocardiography (TOE) according to different streptococcal species. The aim of this sub-study was to propose a flowchart for the use of echocardiography in streptococcal BSIs.

Methods: In a population-based setup, we investigated all patients admitted with streptococcal BSIs and crosslinked data with nationwide registries to identify comorbidities and concomitant hospitalization with IE. Streptococcal species were divided in four groups based on the crude risk of being diagnosed with IE (low-risk < 3\%, moderate-risk 3-10\%, high-risk 10-30\% and very high-risk > 30\%). Based on number of positive blood culture (BC) bottles and IE risk factors (prosthetic valve, previous IE, native valve disease, and cardiac device), we further stratified cases according to probability of concomitant IE diagnosis to create a flowchart suggesting TTE plus TOE (IE > 10\%), TTE (IE 3-10\%), or "wait \& see" (IE < 3\%).

Results: We included 6393 cases with streptococcal BSIs (mean age 68.1 years [SD 16.2], 52.8\% men). BSIs with lowrisk streptococci (S. pneumoniae, S. pyogenes, S. intermedius) are not initially recommended echocardiography, unless they have $\geq 3$ positive BC bottles and an IE risk factor. Moderate-risk streptococci (S. agalactiae, S. anginosus, S. constellatus, S. dysgalactiae, S. salivarius, S. thermophilus) are guided to "wait \& see" strategy if they neither have a risk factor nor $\geq 3$ positive $B C$ bottles, while a $T E$ is recommended if they have either $\geq 3$ positive $B C$ bottles or a risk factor. Further, a TTE and TOE are recommended if they present with both. High-risk streptococci (S. mitis/oralis, S. parasanguinis, $G$. adiacens) are directed to a TTE if they neither have a risk factor nor $\geq 3$ positive BC bottles, but to TTE and TOE if they have either $\geq 3$ positive BC bottles or a risk factor. Very high-risk streptococci (S. gordonii, S. gallolyticus, S. mutans, S. sanguinis) are guided directly to TTE and TOE due to a high baseline IE prevalence.
\end{abstract}

\footnotetext{
* Correspondence: sanch@regionsjaelland.dk

'Department of Cardiology, Zealand University Hospital, Roskilde, Denmark

${ }^{2}$ Department of Cardiology, Copenhagen University Hospital Herlev and

Gentofte, Hellerup, Denmark

Full list of author information is available at the end of the article
}

C C The Author(s). 2021 Open Access This article is licensed under a Creative Commons Attribution 4.0 International License, which permits use, sharing, adaptation, distribution and reproduction in any medium or format, as long as you give appropriate credit to the original author(s) and the source, provide a link to the Creative Commons licence, and indicate if changes were made. The images or other third party material in this article are included in the article's Creative Commons licence, unless indicated otherwise in a credit line to the material. If material is not included in the article's Creative Commons licence and your intended use is not permitted by statutory regulation or exceeds the permitted use, you will need to obtain permission directly from the copyright holder. To view a copy of this licence, visit http://creativecommons.org/licenses/by/4.0/ The Creative Commons Public Domain Dedication waiver (http://creativecommons.org/publicdomain/zero/1.0/) applies to the data made available in this article, unless otherwise stated in a credit line to the data. 
Conclusion: In addition to the clinical picture, this flowchart based on streptococcal species, number of positive blood culture bottles, and risk factors, can help guide the use of echocardiography in streptococcal bloodstream infections. Since echocardiography results are not available the findings should be confirmed prospectively with the use of systematic echocardiography.

Keywords: Infective endocarditis, Streptococcal species, Bloodstream infection, Echocardiography

\section{Background}

Streptococci are one of the leading causes of infective endocarditis (IE), yet there is uncertainty when to screen for IE in patients with streptococcal bloodstream infections (BSIs) [1-4]. A nationwide registry study found an IE prevalence of $16.7 \%$ in Enterococcus faecalis (E. faecalis), 10.1\% in Staphylococcus aureus (S. aureus), and 7.3\% in streptococcal bloodstream infections (BSIs), leading the authors to suggest that screening for IE in these patients seems reasonable [5]. In both S. aureus and E. faecalis BSIs a more extensive use of echocardiography has been recommended based on echocardiographic screening studies finding a IE prevalence around 15-25\% [68].

In a recent large cohort study, we showed that different streptococcal species had markedly different IE prevalence, ranging from 1 to $2 \%$ in S. pneumoniae and S. pyogenes to almost $50 \%$ in S. gordonii and S. mutans [9]. These findings support the fact that not all streptococcal species carry the same risk of IE and therefore it is highly relevant to consider if the work-up should be differentiated between patients infected with different streptococcal species. The current European and American guidelines on IE are not specifying anything regarding the prevalence of IE nor the differentiation of workup in patients with BSIs due to different streptococcal species $[10,11]$. Furthermore, risk factors for IE in patients with a streptococcal BSI have been sparsely investigated. Sunnerhagen et al. created a score to guide the use of echocardiography in non- $\beta$-haemolytic streptococcal BSIs based on different risk factors [12]. However, the study was limited by low numbers, not differentiating between when to perform transthoracic (TTE) or transoesophageal echocardiography (TOE) and failing to include all the different streptococcal species.

In the present study our aim was to propose a flowchart for the use of echocardiography in patients with streptococcal BSIs based on retrospective data of IE prevalence according to streptococcal species and IE risk factors.

\section{Methods}

\section{Study population, data sources and case definitions}

This is a sub-study to a population-based study including adult patients with monospecies streptococcal BSIs admitted from January 1, 2008 to December 31, 2017 in the Capital Region of Denmark (1.5 million people) [9]. The full method has been described in detail in the main study. In addition to the main study exclusion of cases with unavailable species identification, the current substudy also excluded rare species accounting for $<0.5 \%$ of BSIs. Using Danish nationwide registries [13, 14] we identified IE cases with hospital admissions with primary and secondary ICD-10 codes (I33, I38 and I39.8) and IE was considered associated to the streptococcal BSI, if the positive blood cultures occurred during the IE admission or up to 30 days prior. Since detailed patient chart data including data on echocardiograms are not available in the registries the estimated IE prevalence is based on the assumption that when the hospital doctors diagnose a patient with IE the patient actually has IE and in the same way, when a patient is not diagnosed with IE it is because the patient does not have IE. To reduce the number of false positive IE diagnoses, we required IE cases to have a hospital admission of minimum 14 days unless they died during the first 14 days of admission because this algorithm have been validated with a positive predictive value (PPV) above $90 \%$ for the diagnosis of IE in the Danish registries $[15,16]$. See Supplemental Table 1 for ICD-, procedure- and ATC-codes used in this study.

\section{Design of echocardiography flowchart}

Based on the crude estimated IE prevalence, each streptococcal species was assigned to a risk group: low $(<3 \%)$, moderate (3-10\%), high (10-30\%), and very high (>30\%). After grouping the streptococcal species in risk groups, the IE prevalence was further assessed in subgroups according to number of positive blood culture bottles and presence of conventional IE risk factors (prosthetic valve, previous IE, native valve disease and cardiac device) $[17$, 18]. From these subgroups we decided to use the following cut-offs for the proposed use of TTE and TOE: (i) IE prevalence $>10 \%$, TTE and are recommended (ii) IE prevalence of $3-10 \%$, TTE is recommended (iii) IE prevalence $<3 \%$, "wait \& see" is recommended, meaning that no initial echocardiography is recommended unless there is a strong clinical suspicion of IE.

\section{Statistics}

Baseline data were presented for the four streptococcal risk groups. Categorical data were expressed as numbers 
and percentage (\%), while continuous variables were presented as mean \pm standard deviation (SD). Estimated prevalence of IE was calculated as BSI cases with concomitant IE diagnosis divided by the total number of BSI cases in the total cohort and in the different streptococcal species groups, respectively. The IE prevalence estimate was presented with a 95\% confidence interval (CI). A multivariable logistic regression analysis with the outcome IE diagnosis in BSIs with different streptococcal species, adjusted for age, sex, $\geq 3$ positive blood culture bottles, native valve disease, prosthetic valve, previous IE, and cardiac device, was carried out with $S$. pneumoniae as a reference species. Results were presented as odds ratio (OR) with 95\% CI. The IE prevalence estimate of every step in the flowchart was calculated as cases with an IE diagnosis divided by the total number of BSI cases in each subgroup, with $<$ or $\geq 3$ positive blood culture bottles, and presence vs. absence of an IE risk factor. IE prevalence estimates were presented with a 95\% confidence interval (CI). All statistical analyses were performed using the SAS statistical software, version 9.4 (SAS Institute, Inc., Cary, NC, USA).

\section{Results}

Patient inclusion and characteristics

From a total of 7122 streptococcal BSI cases we identified 6506 eligible cases with monospecies streptococcal BSIs in 6224 unique patients, of which 236 patients had more than one BSI case with a median of 332 days [IQR 102-869] between the first and the second episode. After excluding rare species (causing $<0.5 \%$ of BSIs) we included 6393 cases with monospecies streptococcal BSIs of which 451 were diagnosed with IE (7.1\%) (Fig. 1). Based on the IE prevalence streptococcal species were classified as low-risk, moderate-risk, high-risk and very high-risk streptococci as described in the main study [9]. There were 3230 BSI cases (51\%) in the low-risk group, 2106 BSI cases (33\%) in the moderate-risk group, 556 BSI cases (9\%) in the high-risk group, and 501 BSI cases (8\%) in the very high-risk group (Fig. 1). In the total cohort the mean age was 68.1 years (SD 16.2) and 52.8\% were men. Baseline characteristics according to risk groups are shown in Table 1 . Cases in the very high-risk streptococci group were older and had the highest rate of native valve disease (13.2\%), prosthetic valve (12.4\%), previous IE (4.4\%), and cardiac device (9.2\%) (Table 1).

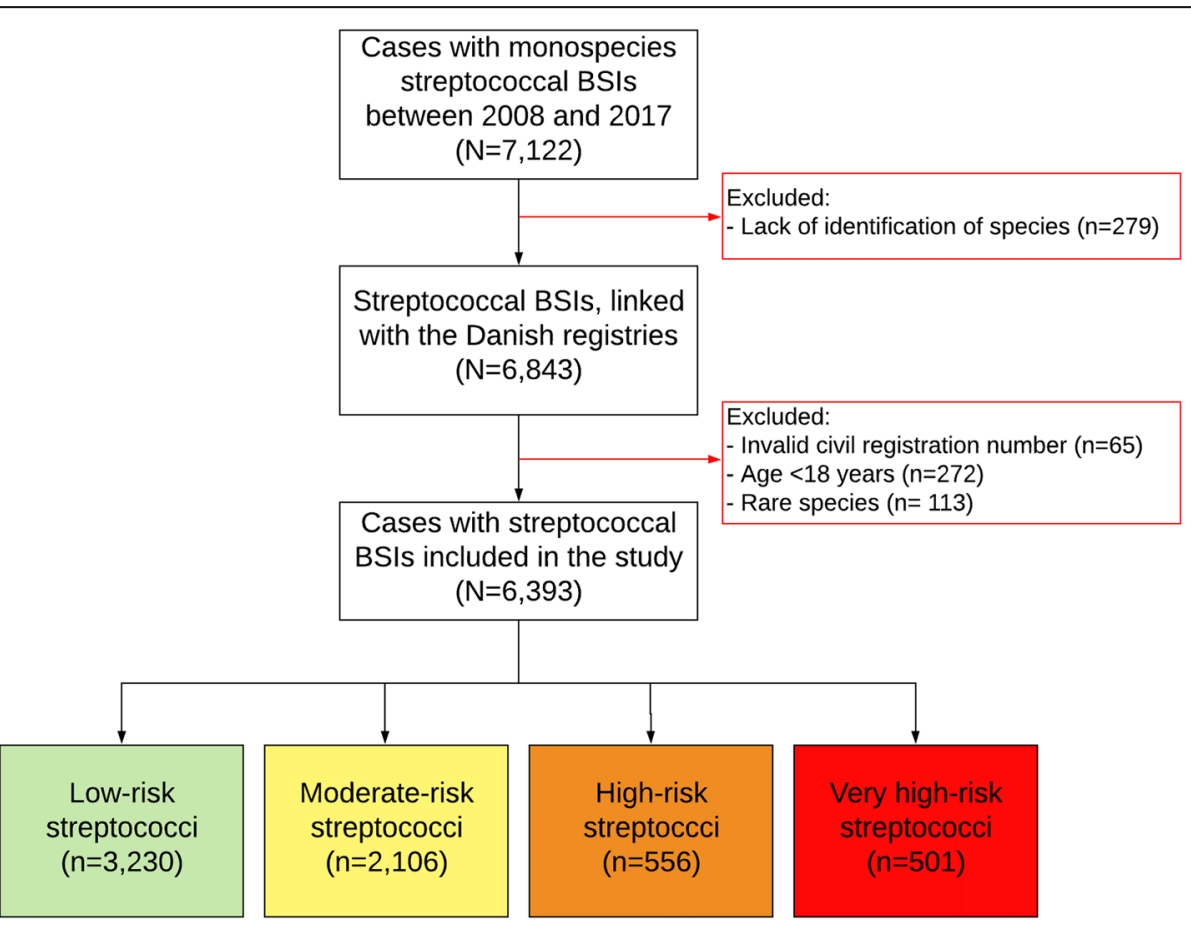

Fig. 1 Patient inclusion. The figure illustrates a flowchart of patient selection. A total of 7122 cases of monospecies streptococcal BSIs were identified. Classification of species identification was unavailable in 279 cases. From the remaining 6843 cases, 65 cases had an invalid civil registration number, 272 cases were below 18 years of age, and 113 cases were infected with rare streptococcal species. BSI, bloodstream infection. Green: low-risk species (IE prevalence <3\%), yellow: moderate-risk species (IE prevalence 3-10\%), orange: high-risk species (IE prevalence 10-30\%), red: very high-risk species (IE prevalence $>30 \%$ ) 
Table 1 Baseline characteristics

\begin{tabular}{|c|c|c|c|c|c|}
\hline & $\begin{array}{l}\text { Low-risk } \\
\text { Streptococci } \\
(n=3230)\end{array}$ & $\begin{array}{l}\text { Moderate-risk } \\
\text { streptococci } \\
(n=2106)\end{array}$ & $\begin{array}{l}\text { High-risk } \\
\text { streptococci } \\
(n=556)\end{array}$ & $\begin{array}{l}\text { Very high-risk } \\
\text { streptococci } \\
(n=501)\end{array}$ & $\begin{array}{l}\text { All BSIs } \\
(N= \\
6393)\end{array}$ \\
\hline \multicolumn{6}{|l|}{ Demographics } \\
\hline Age, mean (SD) & $66.8(16.5)$ & $69.4(15.6)$ & $67.0(17.1)$ & $72.2(14.2)$ & $68.1(16.2)$ \\
\hline Male, n (\%) & $1543(47.8)$ & $1204(57.2)$ & $325(58.5)$ & $301(60.1)$ & $\begin{array}{l}3373 \\
(52.8)\end{array}$ \\
\hline \multicolumn{6}{|l|}{ Comorbidities $^{a}$} \\
\hline $\begin{array}{l}\text { Native valve disease }{ }^{\mathrm{b}}, \mathrm{n} \\
(\%)\end{array}$ & $97(3.0)$ & $106(5.0)$ & $38(6.8)$ & $66(13.2)$ & $307(4.8)$ \\
\hline Prosthetic valve, n (\%) & $47(1.5)$ & $61(2.9)$ & $28(5.0)$ & $62(12.4)$ & $198(3.1)$ \\
\hline Previous IE, n (\%) & $23(0.7)$ & $34(1.6)$ & $12(2.2)$ & $22(4.4)$ & $91(1.4)$ \\
\hline Cardiac device ${ }^{c}, \mathrm{n}(\%)$ & $120(3.7)$ & $101(4.8)$ & $26(4.7)$ & $46(9.2)$ & $293(4.6)$ \\
\hline IHD, n (\%) & $478(14.8)$ & $428(20.3)$ & $128(23.0)$ & $138(27.5)$ & $\begin{array}{l}1172 \\
(18.3)\end{array}$ \\
\hline $\mathrm{CHF}, \mathrm{n}(\%)$ & $365(11.3)$ & $347(16.5)$ & 89 (16.0) & $123(24.6)$ & $924(14.5)$ \\
\hline Cancer, n (\%) & $787(24.4)$ & $671(31.9)$ & $162(29.1)$ & $130(25.9)$ & $\begin{array}{l}1750 \\
(27.4)\end{array}$ \\
\hline COPD, n (\%) & $612(18.9)$ & $280(13.3)$ & $105(18.9)$ & $86(17.2)$ & $\begin{array}{l}1083 \\
(16.9)\end{array}$ \\
\hline $\mathrm{DM}, \mathrm{n}(\%)$ & $386(12.0)$ & $372(17.7)$ & $84(15.1)$ & $101(20.2)$ & $943(14.8)$ \\
\hline Renal disease, n (\%) & $230(7.1)$ & $209(9.9)$ & $64(11.5)$ & $57(11.4)$ & $560(8.8)$ \\
\hline Renal dialysis, n (\%) & $44(1.4)$ & $40(1.9)$ & $13(2.3)$ & $15(3.0)$ & $112(1.8)$ \\
\hline
\end{tabular}

BSI Bloodstream infection, CHF Congestive heart failure, COPD Chronic obstructive pulmonary disease, DM Diabetes mellitus, IE Infective endocarditis, IHD Ischemic heart disease

${ }^{a}$ medical history prior to $\mathrm{IE}{ }^{\mathrm{b}}$ Native valve disease without prosthetic valve, ${ }^{c}$ cardiac implantable electronic device

\section{Adjusted risk of infective endocarditis}

Figure 2 shows the crude prevalence of IE according to streptococcal species and the multivariable logistic regression adjusted for age, sex, and IE risk factors $(\geq 3$ positive blood culture bottles, native valve disease, prosthetic valve, previous IE and cardiac device). In the adjusted analysis including a slightly smaller cohort than in the main study, the overall risk pattern remained stable with progressively higher associated risk of IE in the species with the higher IE prevalence (Fig. 2).

\section{Endocarditis prevalence according to species, number of positive blood culture bottles, and risk factors}

The IE prevalence was calculated for each streptococcal risk group and is presented relative to the number of positive blood culture bottles, and IE risk factors (Table 2). Low-risk streptococci (S. pneumoniae, S. pyogenes, and S. intermedius) had an IE prevalence of $0.5-1.5 \%$, unless they had $\geq 3$ positive blood culture bottles and at least one further risk factor in which case IE prevalence was 6.9\% [95\% CI: 3.4-12.3\%]. Moderate-risk streptococci (S. agalactiae, S. anginosus, S. constellatus, S. dysgalactiae, S. salivarius, and $S$. thermophilus) had an IE prevalence of 1.5\% [95\% CI: 0.9-2.4\%] if they neither had a risk factor nor $\geq 3$ positive blood culture bottles, but an IE prevalence of
$3-10 \%$ if they had either $\geq 3$ positive blood culture bottles (IE prevalence 9.6\% [95\% CI: 7.5-12.1\%]) or a risk factor (IE prevalence 8.7\% [95\% CI: 4.4-15.1\%]). Moderate-risk streptococci presenting with both $\geq 3$ positive blood culture bottles and a risk factor had an IE prevalence of $31.1 \%$ [95\% CI: 23.3-39.7\%]. Highrisk streptococci (S. mitis/oralis, S. parasanguinis, and G. adiacens) had an IE prevalence of 3.6\% [95\% CI: 1.9-6.0\%]) if they neither had a risk factor nor $\geq 3$ positive blood culture bottles, but an IE prevalence of 14.0\% [95\% CI: 5.3-27.9\%] if they presented a risk factor and $<3$ positive blood culture bottles and an IE prevalence of $48.4 \%$ [95\% CI: 40.4-56.5\%] if they had $\geq 3$ positive blood culture bottles. Finally, very highrisk streptococci (S. gordonii, S. gallolyticus (formerly S. bovis), S. mutans, and S. sanguinis) had a very high baseline IE prevalence of $35.7 \%$ [95\% CI: 31.5-40.1\%] (Table 2).

\section{Proposed flowchart for echocardiography in streptococcal bacteraemia}

Based on the estimated IE prevalence in BSIs with different streptococcal species and taking the number of positive blood culture bottles and IE risk factors into account we designed a flowchart for the use of TTE and TOE (Fig. 3). Using the specified IE prevalence cut-offs, 


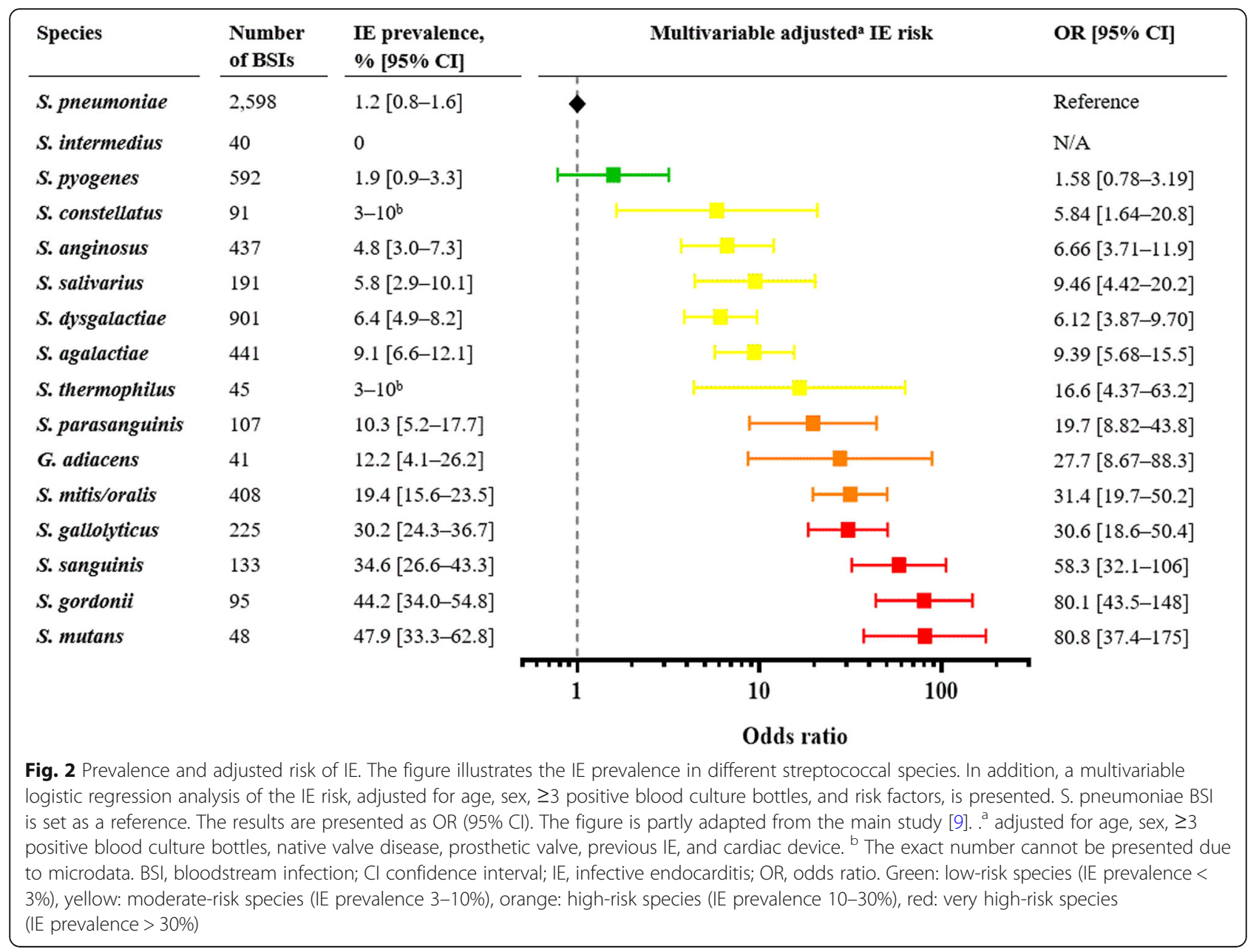

Table 2 IE prevalence for steps in the flowchart

\begin{tabular}{llll}
\hline & Number of BSIs & Number of IE cases & IE prevalence with [95\% CI] \\
\hline Low-risk species, baseline & 3230 & 41 & $1.3 \%[0.9-1.7 \%]$ \\
$<3$ positive BC bottles & 1500 & 7 & $0.5 \%[0.2-1.0 \%]$ \\
$\geq 3$ positive BC bottles without risk factors & 1585 & 24 & $1.5 \%[1.0-2.2 \%]$ \\
$\geq 3$ positive BC bottles with a risk factor & 145 & 10 & $6.9 \%[3.4-12.3 \%]$ \\
Moderate-risk species, baseline & 2106 & 136 & $6.5 \%[5.4-7.6 \%]$ \\
$<3$ positive BC bottles without risk factors & 1163 & 18 & $1.5 \%[0.9-2.4 \%]$ \\
$<3$ positive BC bottles with a risk factor & 126 & 11 & $8.7 \%[4.4-15.1 \%]$ \\
$\geq 3$ positive BC bottles without risk factors & 685 & 66 & $9.6 \%[7.5-12.1 \%]$ \\
$\geq 3$ positive BC bottles with a risk factor & 132 & 41 & $31.1 \%[23.3-39.7 \%]$ \\
High-risk species, baseline & 556 & 95 & $17.1 \%[14.0-20.5 \%]$ \\
$<3$ positive BC bottles without risk factors & 356 & 13 & $3.6 \%[1.9-6.0 \%]$ \\
$<3$ positive BC bottles with a risk factor & 43 & 6 & $14.0 \%[5.3-27.9 \%]$ \\
$\geq 3$ positive BC bottles & 157 & 76 & $48.4 \%[40.4-56.5 \%]$ \\
Very high-risk species, baseline & 501 & 179 & $35.7 \%[31.5-40.1 \%]$ \\
\hline
\end{tabular}

$B C$ Blood culture, $B S I$ Bloodstream infection, $C I$ Confidence interval, IE Infective endocarditis Risk factors: native valve disease, prosthetic valve, previous IE or cardiac device 


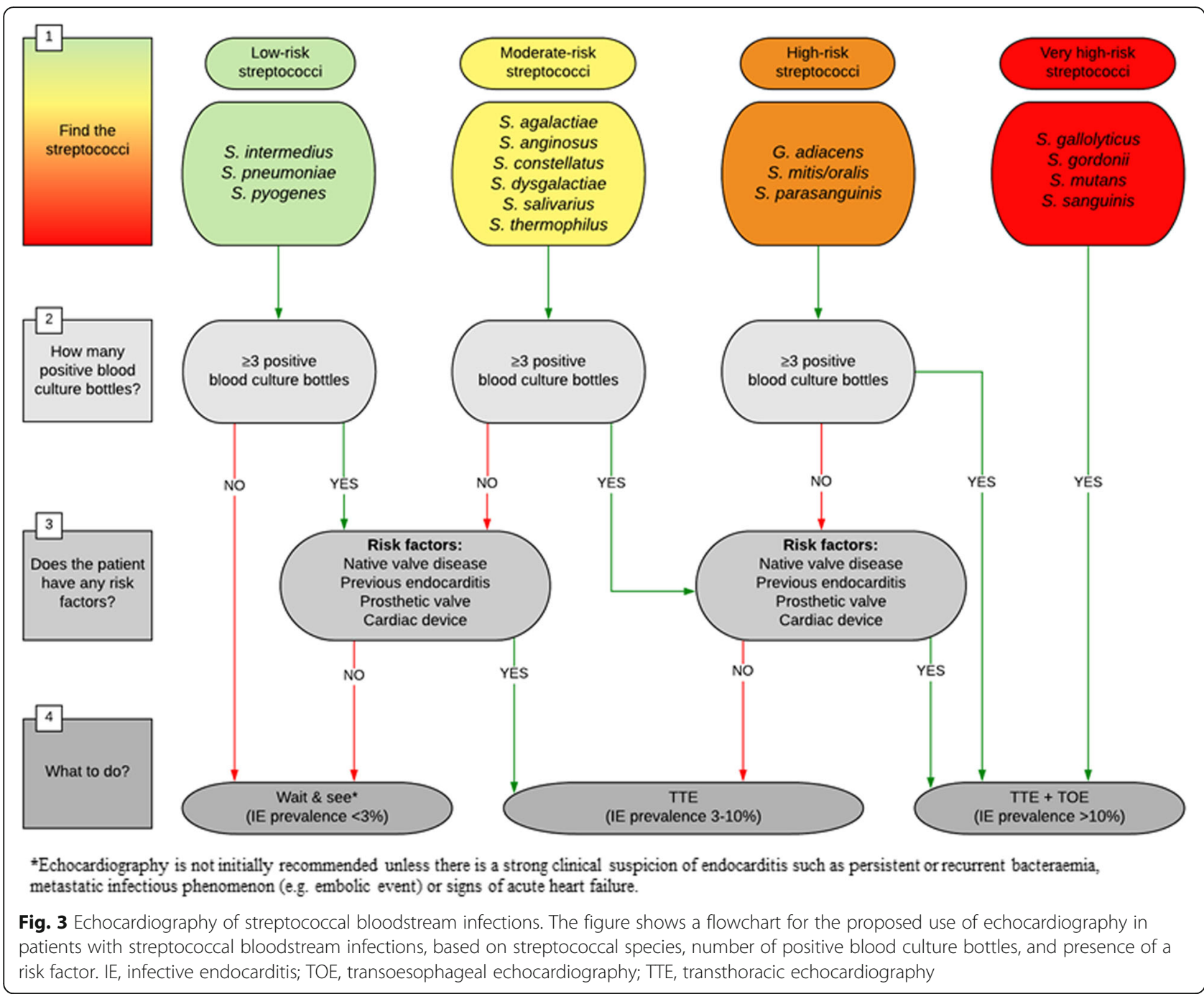

we propose the following strategy: "wait \& see" (IE prevalence <3\%), TTE (IE prevalence 3-10\%), and TTE plus TOE (IE prevalence $>10 \%$ ). In this way, low-risk streptococci are directed to "wait \& see", unless they have $\geq 3$ positive blood culture bottles and an IE risk factor in which case they are led to TTE. Moderate-risk streptococci with $<3$ positive blood culture bottles and no risk factors are guided to "wait \& see", whereas a TTE is recommended if they have either $\geq 3$ positive blood culture bottles or a risk factor. However, moderate-risk streptococci presenting with both $\geq 3$ positive blood culture bottles and a risk factor are recommended a TTE plus TOE. High-risk streptococci are recommended a TTE if they neither have a risk factor nor $\geq 3$ positive blood culture bottles, but a TTE plus TOE if they have either $\geq 3$ positive blood culture bottles or a risk factor. Finally, very high-risk streptococci are recommended a TTE plus TOE due to a very high baseline IE prevalence.

\section{Discussion}

Based on estimated IE prevalence, this study proposes a flowchart for the use of echocardiography in patients with BSIs caused by different streptococcal species. The main findings were: (i) BSI cases with a low-risk streptococcus (S. pneumoniae, S. pyogenes or S. intermedius) are not initially being recommended an echocardiography, unless they have $\geq 3$ positive blood culture bottles and presence of an IE risk factor (ii) BSI cases with a very high-risk streptococcus (S. gordonii, S. gallolyticus, $S$. mutans, and $S$. sanguinis) or cases with $\geq 3$ positive blood culture bottles with a high-risk streptococcus $(S$. mitis/oralis, S. parasanguinis or G. adiacens) are recommended to a TTE plus TOE.

To evaluate the possible clinical implications of these findings it is relevant to discuss the current clinical guidelines deciding when to perform TTE and/or TOE in patients with streptococcal BSIs. Neither IE guidelines from the European Society of Cardiology (ESC) nor the American Heart Association (AHA), are specifying 
anything regarding differentiation of work-up in patients with BSI due to different streptococcal species $[10,11]$. However, in the diagnostic criteria for IE, which are based on the modified Duke criteria, BSIs with viridans streptococci and S. bovis are major diagnostic criteria $[10,19]$. It is concerning that the term viridans streptococci covers all species in the $S$. anginosus group, $S$. mitis group, $S$. mutans group, and $S$. salivarius group without any distinction between specific species [20]. It is now evident, that different streptococcal species within the so called viridans streptococci have different IE prevalence, ranging from $4.8 \%$ in S. anginosus to $47.9 \%$ in S. mutans [9]. Therefore, it is highly relevant to differentiate the work-up in patients with streptococcal BSIs with different streptococcal species based on the associated risk of IE. To confront this problem, Sunnerhagen et al. created the HANDOC score based on 339 non- $\beta$-haemolytic streptococcal BSI cases and 26 cases of IE [12]. The score is aimed to guide the use of echocardiography by evaluating six factors: Heart murmur or valvular disease, Aetiology, Number of cultures, Duration of symptoms, Only one species, and Community acquisition. Despite the fact, that the score showed a high sensitivity in an external validation [21], the study was limited by only including few IE cases, not including all the different streptococcal species and not differentiating between when to perform TTE or TOE. Surprisingly, the authors only found 4 cases of IE in 102 S. mitis group BSIs in sharp contrast to most other studies finding $S$. mitis to cause the majority of IE cases in streptococcal BSIs $[9,22,23]$. Further, the authors suggest a reduction in the calculated score when patients are infected with $S$. anginosus BSIs, since they did not find any IE cases in 105 BSIs with S. anginosus [12]. This finding is not in line with recent findings in a much larger cohort, where the IE prevalence for S. anginosus BSIs was almost 5\% (21 of 431) [9].

With nearly 6400 streptococcal BSI cases, we had sufficient numbers to evaluate the prevalence of IE on species level and stratify the species according to the prevalence of IE into low, moderate, high, and very high risk of IE. The overall estimated IE prevalence in our cohort $(7.1 \%)$ was not markedly different from the one found in non- $\beta$-haemolytic streptococcal BSI (7.7\%) and mixed streptococcal BSI (10.6\%) [12, 24]. In addition, using the information from earlier studies of IE risk factors such as native valve disease, prosthetic valve, previous IE, and cardiac device we were able to incorporate these details in the flowchart $[17,18]$. To determine the IE prevalence cut-offs for our proposed use of echocardiography we combined clinical experience with knowledge from earlier studies on typical IE bacteria to reach a consensus decision. Previous studies on S. aureus and E. faecalis IE have recommended TTE and TOE based on IE prevalence from 10 to $25 \%$ [5-7]. We decided to use an IE prevalence of $10 \%$ as cut-off for TOE to accommodate the fact that our numbers are likely to be conservative estimates lacking information on BSI cases where no echocardiography was performed (data not available). To select the IE prevalence limit for an expectant strategy ("wait \& see") we considered the prevalence of IE in S. aureus and E. faecalis BSI cases without any risk factors $(3.4-5 \%)$ from earlier studies [6, 7]. Since these patients in low to moderate risk of IE are often still recommended a TTE we chose a lower limit of $<3 \%$ for the expectant strategy. In our study the IE prevalence in the subgroups of BSI cases leading to a "wait \& see" strategy was well below this limit. It is adamant to underscore that the flowchart is thought as an additional tool to help the clinician and that the overall clinical assessment of the patient is still crucial. Therefore, clinical findings such as persistent or recurrent bacteraemia, signs of metastatic infection (e.g. embolic event) or acute heart failure should of course lead to echocardiography and work up for IE no matter the outcome of the flowchart.

\section{Limitations}

The flowchart is created on retrospective data from a partly register-based setup, which naturally introduces limitations. Firstly, the diagnosis of IE was based on ICD-10 discharge codes with the inherent risk of misclassification bias. However, the applied method using ICD-10 codes in the Danish registries has been validated with a positive predictive value for the diagnosis of IE of $90 \%$ [15]. Since the negative predictive value of the IE diagnosis in the Danish registries has not been investigated, we cannot accurately estimate the amount of overlooked IE patients that were misclassified (false negatives) by not receiving an ICD-10 IE diagnosis. Secondly, since data on TTE and TOE are not available, the flowchart was created retrospectively without knowledge of the use of echocardiography in the different groups. Since echocardiography plays a central role in diagnosing IE it would have been relevant to know if some species were less often examined with echocardiography thereby increasing the probability of ascertainment bias and missed IE cases. Having that in mind, the IE prevalence should be interpreted as conservative estimates. At the same time, the missing data on echocardiograms introduces a risk of circular deduction where the species more typically examined by echocardiography also becomes the species where IE is diagnosed most often. In other words that the current clinical selection of patients for echocardiography are already performed in a manner parallel to the suggested flow chart without collective consciousness of the specific selection process. In this way the suggested flowchart may just be the first actual 
description of the performed clinical practice applied by IE experts. The only way to answer this question is to perform a prospective screening study with systematic echocardiography in all patients with streptococcal BSI. Thirdly, all positive $\mathrm{BC}$ bottles occurring within 14 days of the first positive $\mathrm{BC}$ were considered part of same BSI episode without the possibility to distinguish persistent bacteraemia from several positive BCs obtained in a single initial BC sampling. Fourth, the present study was performed in a specific geographical setting in Scandinavia, which may not represent the prevalence of IE according to streptococcal species in other regions of the world. Finally, it is important to emphasize that the proposed flowchart should not stand alone and the clinical evaluation of the patient is still central in the decision making as stated in the international guidelines [10,11].

\section{Conclusion}

In addition to the clinical picture, this flowchart based on streptococcal species, number of positive blood culture bottles, and risk factors, can help guide the decision to perform echocardiography in patients with streptococcal bloodstream infections. Since echocardiography results are not available the flowchart should be further validated in a clinical prospective setup with use of systematic echocardiography and in other geographical settings.

\section{Abbreviations \\ AHA: American Heart Association; ATC: Anatomical Therapeutic Chemical; BC: Blood culture; BSI: Bloodstream infection; CHF: Congestive heart failure; Cl: Confidence interval; COPD: Chronic obstructive pulmonary disease; DM: Diabetes mellitus; E. faecalis: Enterococcus faecalis; ESC: European Society of Cardiology; G. adiacens: Granulicatella adiacens; ICD: International Classification of Diseases; IE: Infective endocarditis; IHD: Ischemic heart disease; NOMESCO: Nordic Medico-Statistical Committee; OR: Odds ratio; PPV: Positive predictive value; S. aureus: Staphylococcus aureus; SD: Standard deviation; TOE: Transoesophageal echocardiography; TTE: Transthoracic echocardiography}

\section{Supplementary Information}

The online version contains supplementary material available at https://doi. org/10.1186/s12879-021-06391-2

Additional file 1: Table S1. ICD-, procedure- and ATC-codes.

\section{Acknowledgements}

Not applicable.

\section{Authors' contributions}

$\mathrm{SCH}, \mathrm{NEB}$ and $\mathrm{AD}$ : Design of the project, analysis of data, draft of the manuscript. MA and JB: Design of the project, data extraction, critical revision of data, reviewing and editing the manuscript. $L \varnothing, E F, L B O, T K L, G G$ and CTP: Design of the project, critical revision of data, reviewing and editing the manuscript. All authors have read and approved the final manuscript.

\section{Funding}

This work was supported by the University Hospital of Copenhagen Herlev and Gentofte (Research Council and Department of Cardiology), Helsefonden, and Director Jacob Madsen and wife Olga Madsen's research foundation. The funders of the study were not involved in the study design, data collection, data analysis, data interpretation, writing of the report, or in the decision to submit the paper for publication.

Availability of data and materials

All supportive data are available within the article and its supplementary files.

\section{Declarations}

\section{Ethics approval and consent to participate}

The study was performed as a retrospective administrative registry study, where all data were anonymised before use. All data used for this study are held by Statistics Denmark, which also has the administrative rights to the data and encrypts the identification number likewise in all datasets. In retrospective register studies in Denmark, ethics approval and individual consent is not required and all methods were performed in accordance with the relevant guidelines and regulations [13]. The data handling and storage in the project has been approved by the Danish Data Protection Agency (approval number 2007-58-015/GEH-2014-018 I-Suite number 02736).

\section{Consent for publication}

Not applicable.

\section{Competing interests}

$\mathrm{SCH}$ has nothing to declare.

NEB has nothing to declare.

$L \varnothing$ has nothing to declare.

MA has nothing to declare.

EF has nothing to declare.

JB has nothing to declare.

LBO has nothing to declare.

TKL has nothing to declare.

GG has nothing to declare.

CTP has nothing to declare.

AD reports grants from The Lundbeck Foundation and the European Society of Cardiology unrelated to this study.

\section{Author details}

'Department of Cardiology, Zealand University Hospital, Roskilde, Denmark. ${ }^{2}$ Department of Cardiology, Copenhagen University Hospital Herlev and Gentofte, Hellerup, Denmark. ${ }^{3}$ Institute of Clinical Medicine, Copenhagen University, Copenhagen, Denmark. ${ }^{4}$ Clinical Institute, Aalborg University, Aalborg, Denmark. ${ }^{5}$ Department of Cardiology, The Heart Centre, Copenhagen University Hospital Rigshospitalet, Copenhagen $\varnothing$, Denmark. ${ }^{6}$ Department of Clinical Microbiology, Copenhagen University Hospital Herlev and Gentofte, Herlev, Denmark. ${ }^{7} T h e$ Danish Heart Foundation, Copenhagen, Denmark. ${ }^{8}$ Department of Cardiology and Clinical Research, Nordsjaellands Hospital, Hillerød, Denmark. ${ }^{9}$ Department of Cardiology, Aalborg University Hospital, Aalborg, Denmark.

Received: 26 January 2021 Accepted: 28 May 2021

Published online: 16 July 2021

\section{References}

1. Habib G, Erba PA, lung B, Donal E, Cosyns B, Laroche C, et al. Clinical presentation, aetiology and outcome of infective endocarditis. Results of the ESC-EORP EURO-ENDO (European infective endocarditis) registry: a prospective cohort study. Eur Heart J. 2019;40(39):3222-32. https://doi.org/1 0.1093/eurheartj/ehz620.

2. Olmos C, Vilacosta I, Fernández-Pérez C, Bernal UL, Ferrera C, García-Arribas $D$, et al. The evolving nature of infective endocarditis in Spain. J Am Coll Cardiol. 2017;70(22):2795-804. https://doi.org/10.1016/j.jacc.2017.10.005.

3. Pant S, Patel NJ, Deshmukh A, Golwala H, Patel N, Badheka A, et al. Trends in infective endocarditis incidence, microbiology, and valve replacement in the United States from 2000 to 2011. J Am Coll Cardiol. 2015:65(19):2070-6. https://doi.org/10.1016/j.jacc.2015.03.518.

4. Erdem H, Puca E, Ruch Y, Santos L, Ghanem-Zoubi N, Argemi X, et al. Portraying infective endocarditis: results of multinational ID-IRI study. Eur J Clin Microbiol Infect Dis. 2019;38(9):1753-63. https://doi.org/10.1007/s10096019-03607-x.

5. Østergaard L, Bruun NE, Voldstedlund M, Arpi M, Andersen C $\varnothing$, Schønheyder $\mathrm{HC}$, et al. Prevalence of infective endocarditis in patients with 
positive blood cultures: a Danish nationwide study. Eur Heart J. 2019;40(39): 3237-44. https://doi.org/10.1093/eurheartj/ehz327.

6. Dahl A, Iversen K, Tonder N, Hoest N, Arpi M, Dalsgaard M, et al. Prevalence of infective endocarditis in enterococcus faecalis bacteremia. J Am Coll Cardiol. 2019;74(2):193-201. https://doi.org/10.1016/j.jacc.2019.04.059.

7. Rasmussen RV, Høst U, Arpi M, Hassager C, Johansen HK, Korup E, et al. Prevalence of infective endocarditis in patients with Staphylococcus aureus bacteraemia: the value of screening with echocardiography. Eur J Echocardiogr. 2011;12(6):414-20. https://doi.org/10.1093/ejechocard/jer023.

8. Dahl A, Lauridsen TK, Arpi M, Sørensen LL, Østergaard C, Sogaard P, et al. Risk factors of endocarditis in patients with enterococcus faecalis bacteremia: external validation of the NOVA score. Clin Infect Dis. 2016;63(6):771-5. https://doi.org/10.1093/cid/ciw383.

9. Chamat-Hedemand S, Dahl A, Østergaard L, Arpi M, Fosbøl E, Boel J, et al. Prevalence of infective endocarditis in streptococcal bloodstream infections is dependent on streptococcal species. Circulation. 2020;142(8):720-30. https://doi.org/10.1161/CIRCULATIONAHA.120.046723.

10. Habib G, Lancellotti P, Antunes MJ, Bongiorni MG, Casalta J-P, Del Zotti F, et al. 2015 ESC guidelines for the management of infective endocarditis: the task force for the Management of Infective Endocarditis of the European Society of Cardiology (ESC) endorsed by: European Association for CardioThoracic Surgery (EACTS), the European Association of Nuclear Medicine (EANM). Eur Heart J. 2015;36(44):3075-128. https://doi.org/10.1093/eurheartj/ ehv319.

11. Baddour LM, Wilson WR, Bayer AS, Fowler VG, Tleyjeh IM, Rybak MJ, et al. Infective endocarditis in adults: diagnosis, antimicrobial therapy, and Management of Complications: a scientific statement for healthcare professionals from the American Heart Association. Circulation. 2015;132(15): 1435-86. https://doi.org/10.1161/CIR.0000000000000296.

12. Sunnerhagen T, Törnell A, Vikbrant M, Nilson B, Rasmussen M. HANDOC: a Handy score to determine the need for echocardiography in non- $\beta$ hemolytic streptococcal bacteremia. Clin Infect Dis. 2018;66(5):693-8. https://doi.org/10.1093/cid/cix880.

13. Schmidt M, Schmidt SAJ, Sandegaard JL, Ehrenstein V, Pedersen L, Sørensen HT. The Danish National Patient Registry: a review of content, data quality, and research potential. Clin Epidemiol. 2015;7:449-90. https://doi.org/10.214 7/CLEP.S91125.

14. Andersen TF, Madsen M, Jørgensen J, Mellemkjoer L, Olsen JH. The Danish National Hospital Register. A valuable source of data for modern health sciences. Dan Med Bull. 1999;46(3):263-8.

15. Østergaard L, Adelborg K, Sundbøll J, Pedersen L, Loldrup Fosbøl E, Schmidt M. Positive predictive value of infective endocarditis in the Danish National Patient Registry: a validation study. Epidemiol Infect. 2018;146(15):1965-7. https://doi.org/10.1017/S0950268818001401.

16. Sundbøll J, Adelborg K, Munch T, Frøslev T, Sørensen HT, Bøtker HE, et al. Positive predictive value of cardiovascular diagnoses in the Danish National Patient Registry: a validation study. BMJ Open. 2016;6(11):e012832. https:// doi.org/10.1136/bmjopen-2016-012832.

17. Østergaard L, Valeur N, Wang A, Bundgaard H, Aslam M, Gislason G, et al. Incidence of infective endocarditis in patients considered at moderate risk. Eur Heart J. 2019:40(17):1355-61. https://doi.org/10.1093/eurheart//ehy629.

18. Østergaard L, Valeur N, Ihlemann N, Bundgaard H, Gislason G, TorpPedersen $C$, et al. Incidence of infective endocarditis among patients considered at high risk. Eur Heart J. 2018;39(7):623-9. https://doi.org/10.1 093/eurheartj/ehx682.

19. Li JS, Sexton DJ, Mick N, Nettles R, Fowler VG, Ryan T, et al. Proposed modifications to the Duke criteria for the diagnosis of infective endocarditis. Clin Infect Dis. 2000;30(4):633-8. https://doi.org/10.1086/313753.

20. Facklam R. What happened to the streptococci: overview of taxonomic and nomenclature changes. Clin Microbiol Rev. 2002;15(4):613-30. https://doi. org/10.1128/CMR.15.4.613-630.2002

21. Sunnerhagen $T$, Andersen MH, Bruun NE, Bundgaard H, Iversen KK, Rasmussen M. External validation of the HANDOC score - high sensitivity to identify patients with non-beta-haemolytic streptococcal endocarditis. Infect Dis. 2019;0:1-4.

22. Kim SL, Gordon SM, Shrestha NK. Distribution of streptococcal groups causing infective endocarditis: a descriptive study. Diagn Microbiol Infect Dis. 2018;91(3):269-72. https://doi.org/10.1016/.diagmicrobio.2018.02.015.

23. Nilson B, Olaison L, Rasmussen M. Clinical presentation of infective endocarditis caused by different groups of non-beta haemolytic streptococci. Eur J Clin Microbiol Infect Dis. 2016;35(2):215-8. https://doi. org/10.1007/s10096-015-2532-5

24. Andersen MH, Holle SLK, Klein CF, Bruun NE, Arpi M, Bundgaard H, et al. Risk for infective endocarditis in bacteremia with gram positive cocci. Infection. 2020;48(6):905-12. https://doi.org/10.1007/s15010-020-01504-6.

\section{Publisher's Note}

Springer Nature remains neutral with regard to jurisdictional claims in published maps and institutional affiliations.
Ready to submit your research? Choose BMC and benefit from:

- fast, convenient online submission

- thorough peer review by experienced researchers in your field

- rapid publication on acceptance

- support for research data, including large and complex data types

- gold Open Access which fosters wider collaboration and increased citations

- maximum visibility for your research: over $100 \mathrm{M}$ website views per year

At BMC, research is always in progress.

Learn more biomedcentral.com/submissions 